\title{
Guidelines for Point-of-Care Fluorescence Imaging for Detection of Wound Bacterial Burden Based on Delphi Consensus
}

\author{
Alisha R. Oropallo ${ }^{1}$, Charles Andersen ${ }^{2}$, Raymond Abdo ${ }^{3}$, Jenny Hurlow ${ }^{4}$, Martha Kelso ${ }^{5}$, Mark Melin ${ }^{6}$ (D) \\ and Thomas E. Serena $7, * \mathbb{D}$ \\ 1 Comprehensive Wound Healing Center and Hyperbarics, Department of Vascular Surgery, Zucker School of \\ Medicine Hofstra/Northwell, Hempstead, NY 11549, USA; aoropallo@northwell.edu \\ 2 Wound Care Clinic, Madigan Army Medical Center Joint Base Lewis-McChord, Renton, WA 98431, USA; \\ charles.a.andersen@civ.mail.mil \\ 3 St. Louis Foot \& Ankle, LLC., St. Louis, MO 63109, USA; raymond.abdo@me.com \\ 4 Consultant Wound Care Specialized Nurse Practitioner, Memphis, TN 37501, USA; jenny.hurlow@gmail.com \\ 5 Wound Care Plus, LLC., Blue Springs, MO 64015, USA; martha.kelos@mywoundcareplus.com \\ 6 M Health Fairview Wound Healing Institute, South Campus, Edina, MN 55435, USA; melinmark7@gmail.com \\ 7 SerenaGroup Research Foundation, 125 Cambridge Park Dr., Cambridge, MA 02140, USA \\ * Correspondence: serena@serenagroups.com; Tel.: +1-617-945-5225
}

Citation: Oropallo, A.R.; Andersen, C.; Abdo, R.; Hurlow, J.; Kelso, M.; Melin, M.; Serena, T.E. Guidelines for Point-of-Care Fluorescence Imaging for Detection of Wound Bacterial Burden Based on Delphi Consensus. Diagnostics 2021, 11, 1219. https:// doi.org/10.3390/diagnostics11071219

Academic Editor: Francesco Sessa

Received: 10 June 2021

Accepted: 29 June 2021

Published: 6 July 2021

Publisher's Note: MDPI stays neutral with regard to jurisdictional claims in published maps and institutional affiliations.

Copyright: (c) 2021 by the authors. Licensee MDPI, Basel, Switzerland. This article is an open access article distributed under the terms and conditions of the Creative Commons Attribution (CC BY) license (https:// creativecommons.org/licenses/by/ $4.0 /)$.

\begin{abstract}
Excessive levels of bacteria impede wound healing and can lead to infectious complications. Unfortunately, clinical signs and symptoms of elevated bacterial burden are often unreliable. As a result, point-of-care fluorescence imaging, used to detect critical bacterial burden in wounds, is becoming widely recognized and adopted by clinicians across the globe as an accepted and added component of wound assessment protocol. A Delphi method was employed to establish consensus guidelines describing fluorescence imaging use. A multidisciplinary panel of 32 wound experts (56\% MD, $22 \%$ podiatrist, $12.5 \%$ nurses/nurse practitioners) representing multiple sites of service (e.g., hospital outpatient, inpatient, private office, long-term care) completed two rounds of online questionnaires. The Delphi included key topics, including competencies required to perform imaging, clinical indications for imaging (e.g., signs/symptoms present, procedures warranting imaging), frequency of imaging, and a clinical workflow algorithm. Describing their clinical experiences of imaging impact, $>80 \%$ reported changes in treatment plans, $96 \%$ reported that imaging-informed treatment plans led to improved wound healing, $78 \%$ reported reduced rates of amputations, and $83 \%$ reported reduced rates of microbiological sampling. The guidelines provided here will help to standardize use of fluorescence imaging among wound care providers and enhance the quality of patient care.
\end{abstract}

Keywords: fluorescence imaging; bacteria; wound care; MolecuLight; Delphi method; consensus; guidelines

\section{Introduction}

Identifying and reducing or eliminating bioburden is fundamental to wound healing. However, the sensitivity of clinical examination is less than $15 \%$ and more than $80 \%$ of wounds with high bacterial loads remain undetected and incompletely treated $[1,2]$. These high levels of bacteria are known to impair wound healing rates [3-6]. As a result of a growing aging population, higher incidence of diabetes, and other chronic disease states, the prevalence of chronic wounds and their associated expenditure continues to rise. Chronic wounds are especially common among the elderly population, who have impaired immune function and frequently fail to mount clinical signs and symptoms of infection (CSS) [7]. 
In the US, Medicare spends USD 96.8 billion annually on chronic wound care [8]; the presence of an infection can further increase these costs by up to $70 \%$ [9]. Elevated loads of bacteria contribute to delayed wound healing, increase the risk of infection, lead to the failure of cellular tissue products (CTPs), and substantially increase the risk of sepsis and amputation [10-15]. A conservative estimate places the financial burden of elevated wound bacteria at more than USD 10 billion in annual Medicare spending. Improved methods of detecting bacterial burden are available and are essential to improving patient outcomes and reducing the significant economic burden of infected wounds.

Point-of-care fluorescence imaging (MolecuLight $i: X^{\mathrm{TM}}$, MolecuLight Inc., Toronto, ON Canada) enables clinicians to noninvasively visualize the presence and location of bacteria at loads $>10^{4} \mathrm{CFU} / \mathrm{g}$. The procedure is performed at the patient's bedside and does not require any patient contact. After preparing the wound (e.g., wiping any blood or debris [16]) and positioning the patient for imaging, a standard image in ambient room light is captured. The room lights are then turned off (or a DarkDrape ${ }^{\circledR}$ is used) to create darkness needed to visualize fluorescence signals (See Figure 1). A light sensor on the device is used to confirm adequate darkness for imaging. The device is then positioned parallel to the wound. A range finder on the fluorescence imaging device ensures appropriate distance from the wound. The violet light on the device is switched on and clinician brings the wound into focus prior to capturing the fluorescence image. These steps may be repeated, as needed, during a single patient encounter. This imaging technology provides a direct, objective, and highly sensitive $[1,17,18]$ method of identifying clinically significant bacterial levels in wounds. Wound care providers can utilize this information to immediately and appropriately manage bacterial burden to improve healing. When illuminated with violet light emitted from the imaging device, porphyrin-producing bacteria produce a red fluorescent signal while Pseudomonas aeruginosa uniquely fluoresces cyan $[19,20]$. These fluorescence signals are captured as point-of-care images or videos, which provide immediate information and documentation on bioburden. Clinical trials using quantitative tissue culture techniques as a reference standard demonstrate a high diagnostic accuracy of fluorescence imaging: $>95 \%$ positive predictive value of red and cyan fluorescence in detecting bacterial loads known to delay healing $[1,20,21]$, and a sensitivity that is $300-400 \%$ higher than the current standard of care assessment [1,2]. Clinical studies in "real-world" settings report improvements in wound healing, reduced amputation rates, and reduced need for antimicrobials and antibiotics (demonstrating improved antibiotic stewardship) when fluorescence imaging is incorporated into the care pathway $[17,22,23]$. Knowledge of bacteria location and load reveals the underappreciated impact of increased levels of bacteria in contributing to and driving wound chronicity [24].

The current study was developed for both clinicians and policy makers in a concerted effort to: (1) establish evidence-based universal guidelines describing the clinical indications for use of fluorescence imaging and (2) develop a prescribed clinical workflow demonstrating how to incorporate the fluorescence imaging procedure into standard of care to inform clinical decision making. 

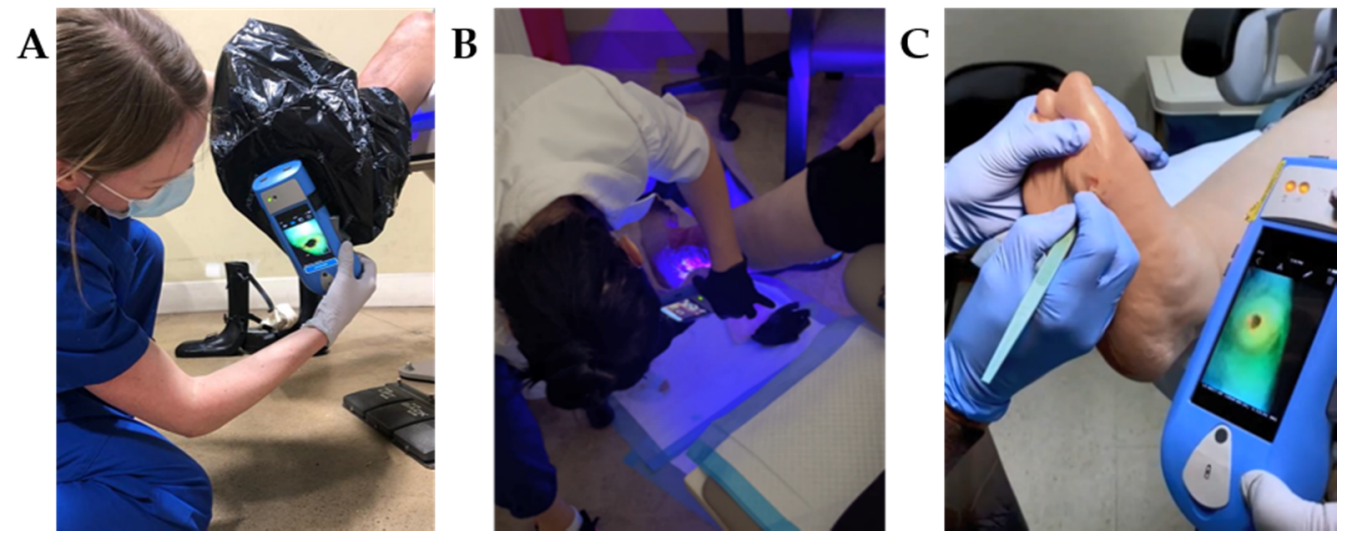

Figure 1. Fluorescence imaging procedure for wound bacterial presence, location and load performed across multiple sites of service. (A) When in a bright environment, a DarkDrape ${ }^{\circledR}$ is attached and used to create adequate darkness. The drape is positioned around the anatomy and the fluorescence imaging device is positioned at an appropriate distance $(8-12 \mathrm{~cm})$ from the wound prior to focusing and capturing an image. (B) The DarkDrape ${ }^{\circledR}$ is not required in a darkened environment. For large wounds wrapping around the anatomy, as shown here, multiple fields of view will be imaged and interpreted. (C) Fluorescence images can be immediately interpreted at the point-of-care to inform treatment. Multiple images may be acquired per visit to assess efficacy of treatments performed. This panel shows the image informing a physician's debridement.

\section{Materials and Methods}

The Delphi technique is a well-established iterative, multi-round process used to collect knowledge and achieve expert consensus on a particular topic through at least two rounds of anonymous surveys [25]. This scientific method has been previously used by wound care experts to establish consensus on guidelines for NPWT with instillation [26], the use of dressings in chronic wound management [27] and development of clinical criteria for infection [28]. As part of the Delphi, experts on a particular topic are invited to put forward opinions in the first round and then indicate their extent of agreement or disagreement on aggregate data in subsequent rounds. This approach is repeated until an accord is established and summarized [29]. The advantages of this method include the ability to involve a large, international group of participants, maintain anonymity of respondents, and the capability of achieving consensus from experts on content informed by clinical evidence $[30,31]$.

\subsection{Expert Panel}

Thirty-nine wound care experts, chosen to reflect various professional groups across key sites of service where wound care is performed, were invited to participate in this independent, unsponsored Delphi consensus. All participants had firsthand experience with the technology. Two weeks prior to sending out the questionnaires, participants were recruited and were informed of the objectives of the Delphi process. Participant responses remained anonymous; individual responses were only known by the study moderator (TS). A subgroup of seven clinician experts formed the core expert panel involved in development of statements and a clinical algorithm (5 to 10 experts are considered adequate for content validation) [25]. Each core expert panel member had more than 15 years of experience providing wound care, and significant expertise using the fluorescence imaging procedure across multiple sites of service.

\subsection{Delphi Survey}

For Round 1, 80 statements were prepared on clinical indications and frequency of performing the fluorescence imaging procedure. These statements were developed based on a review of the literature in combination with the clinical experience of the core expert panel. Participants were asked to select statements that, in their opinion, best reflected 
the clinical indications for use of fluorescence imaging. Participants were also asked to rate their agreement on a clinical workflow describing fluorescence imaging. A space was provided below each question to encourage participants to suggest additional criteria they felt were relevant to fluorescence imaging and its workflow, or to provide clarification on the items they selected. In Round 1, demographic information was also collected (licensed profession, years of experience, and duration of using the imaging device in their practice). In Round 2, responses from Round 1 that achieved consensus ( $>75 \%)$ were aggregated together focusing on various aspects of the imaging procedure (i.e., conditions or procedures warranting use of imaging, etc.) and participants were asked to review a revised clinical workflow diagram incorporating recommendations collected in Round 1. In Round 2, participants were also asked to report the real-world impact of imaging on their own patients' wound outcomes by indicating their agreement using a 4-point Likert scale ranging from "strongly agree" to "strongly disagree". Participants had the option of selecting "unable to comment" if they felt they lacked sufficient experience with the device to answer the question.

\section{Results \& Discussion}

\subsection{Delphi Participants}

Invitations to voluntarily participate in the Delphi survey were sent by email to 39 wound care experts; a response was received from 32 experts, all of whom accepted the invitation. All experts participating were health care professionals providing wound care with $>3$ months experience using the fluorescence imaging device. Of the 32 healthcare professionals who participated in the Delphi, $84 \%$ were from the US, $78 \%$ were physicians and $63 \%$ had $>15$ years of experience in wound care (Table 1). Most participants worked in the hospital outpatient department $(75 \%)$, inpatient department $(62.5 \%)$, a private office $(53.1 \%)$, and/or a long term care facility (18.8\%) settings.

Table 1. Participant Demographics.

\begin{tabular}{|c|c|c|c|}
\hline \multicolumn{2}{|l|}{ Country } & \multicolumn{2}{|l|}{ Licensed Profession } \\
\hline USA & $84.0 \%$ & Medical doctor & $56.3 \%$ \\
\hline Canada & $6.3 \%$ & Podiatrist & $21.9 \%$ \\
\hline United Kingdom & $9.4 \%$ & Nurse Practitioner & $9.4 \%$ \\
\hline & & Physical Therapist & $3.1 \%$ \\
\hline Sites of Service & & Nurse & $9.4 \%$ \\
\hline Hospital Outpatient & $75.0 \%$ & & \\
\hline Hospital Inpatient & $62.5 \%$ & Years of Experience in wound care & \\
\hline Private Office & $53.1 \%$ & $>20$ & $40.6 \%$ \\
\hline Telehealth & $34.4 \%$ & 15 to 20 & $21.9 \%$ \\
\hline Long Term Care Facility & $18.8 \%$ & 10 to 15 & $25.0 \%$ \\
\hline Long Term Acute Care Hospital & $15.6 \%$ & 5 to 10 & $6.3 \%$ \\
\hline Home Health & $9.4 \%$ & 0 to 5 & $6.3 \%$ \\
\hline Other (i.e., Mobile Unit, Urgent Care) & $37.5 \%$ & & \\
\hline
\end{tabular}

\subsection{Delphi Results}

$82 \%$ of invitees completed Round 1 (32/39) and $97 \%$ of clinicians who participated in Round 1 completed Round 2 (31/32), producing an average response rate of $89 \%$. All initiated surveys had 100\% completion for both rounds. In Round 1, 41 out of 80 statements reached consensus. Of the remaining 39 statements, 9 were near consensus (50-75\%) and 30 items did not reach consensus ( $<50 \%$ agreement). In Round 2; statements that achieved or were close to consensus $(>50 \%)$ were aggregated together to produce a total of 14 summary statements. $100 \%$ consensus was achieved for 4 of these summary statements. 


\subsection{Recommendations}

\subsubsection{Competencies Required to Perform Fluorescence Imaging}

The competencies required to perform this procedure are unique and are not currently available through professional programs or courses. These competencies range from fundamental to advanced skills related to setting up the patient and device, how to use the device and how to interpret images. Competence in both fundamental and advanced skills are required to successfully integrate fluorescence-based information on bacterial load into treatment planning. This is especially true for image interpretation, which has a learning curve that improves over time. An online educational program with didactic and hands-on components is offered to new users prior to implementing the procedure in their clinical practice. This includes a free, interactive e-learning module on image interpretation through which clinicians can receive certification.

Most experts agreed that these training components were necessary or critical to perform the imaging procedure, with consensus ranging between $93-100 \%$ after Round 1 for items listed in Table 2. This level of agreement suggests that the experts felt strongly that these competencies were necessary to effectively perform the imaging procedure.

Table 2. Competencies mandatory to perform fluorescence imaging of bacterial burden.

- Setting up device (imaging in focus, download software, exporting images)

- Capturing images (focusing)

- Understanding range finder and light indicators

- How to position and distance the patient and device appropriately

- Adequate lighting conditions ${ }^{1}$
- How to interpret images with red fluorescence

- How to interpret images with cyan fluorescence
- Using image interpretation to plan treatment

- Aligning image interpretation to location of elevated levels of bacteria

\footnotetext{
${ }^{1}$ Darkness is required and achieved by turning off the lights or using a DarkDrape.
}

\subsubsection{Fluorescence Imaging Clinical Workflow}

A clinical workflow (Figure 2) algorithm, based on prior publications demonstrating the benefit of similar fluorescence workflows in promoting healing [23,24], was developed. Iterative loops are a key component of the algorithm: images provide real-time feedback and assist in determining the effectiveness of wound care procedures such as cleansing and debridement. The clinical actions in the workflow and the multiple opportunities for assessment and interpretation enable clinicians to provide comprehensive care.

Respondents agreed with the processes included in the clinical algorithm, with $81.3 \%$ of respondents indicating 'agree' or 'strongly agree' after Round 1. After Round 2, 93.5\% of respondents indicated agreement. Many experts commented that the sequence of steps outlined in the workflow accurately represented their decision-making process and that fluorescence imaging "provides important information guiding clinical management decisions and testing". Specifically, one respondent indicated "this workflow includes everything needed in the plan of care for a patient from assessment to education".

All participants agreed that the clinical algorithm described in Figure 2 may be used on a variety of wound types. There was $100 \%$ agreement among all 32 experts on use of this imaging procedure to assess diabetic foot ulcers. Similarly, high levels of agreement $(80-97 \%)$ were obtained for other common chronic and acute wounds (Table 3). In addition to this list, there are less commonly occurring wounds (e.g., pilonidal sinus, pyoderma gangrenosum) for which a point-of-care diagnosis of bacterial presence and location, or absence, would have high clinical value [32,33]. 


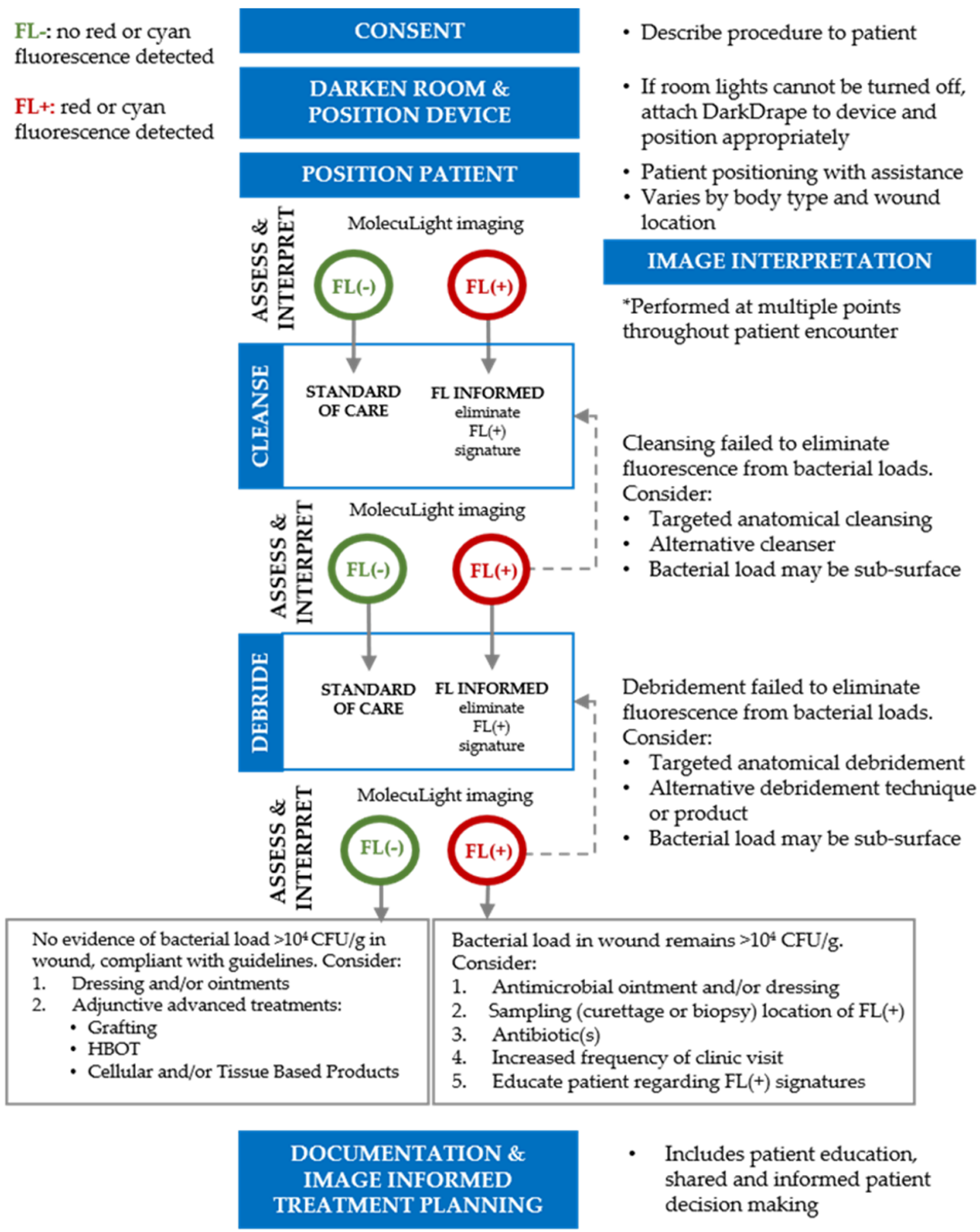

Figure 2. Clinical workflow for point-of-care fluorescence imaging. This clinical workflow describes the typical processes performed by clinicians when incorporating fluorescence imaging into their care pathway. This iterative approach provides clinicians with evidence of bacterial burden at the pointof-care and emphasizes use of clinical judgement to select actions that target removal of bacterial burden from the wound to facilitate wound healing. FL, fluorescence imaging; HBOT, hyperbaric oxygen therapy.

Table 3. Wound types where fluorescence imaging of bacterial burden may be indicated.

Fluorescence Imaging May Be Performed on (but Not Limited to) Any of the Following Wound Types:

Diabetic foot ulcer

Venous leg Ulcer

Pressure Ulcer

Surgical Site infection

Post-operative wound

Traumatic wound 


\subsubsection{Clinical Indications for Fluorescence Imaging to Detect Bacterial Burden in Wounds}

The clinical indications for using fluorescence imaging were divided into 4 areas: (1) review patient medical history/prior treatment, (2) clinical assessment, (3) provision of interventions/treatments and (4) wound sampling. Experts agreed that the criteria for patient history and comorbidities warranted the use of fluorescence imaging. For example, it is well known that comorbid medical conditions, such as diabetes, can mask the presence of signs and symptoms of infection [11,34], and there is a strong association between delayed wound healing and presence of high bacterial burden $[4,5,10,35]$. The $100 \%$ consensus achieved for the patient history and comorbidity criteria suggests that clinicians strongly consider these criteria when deciding to perform fluorescence imaging.

Previous studies comparing fluorescence imaging to standard of care observed a high likelihood of elevated bacterial loads in wounds with few or no clinical signs or symptoms of infection [1,2]; therefore, the panel chose, with $96.7 \%$ agreement, that only one sign or symptom of infection needed to be present to indicate the need for imaging. However, it is not mandatory that any signs and symptoms be present, given that assessment of clinical signs and symptoms may miss the detection of $>80 \%$ of wounds harboring high bacterial loads $[1,3]$.

Point-of-care selection and monitoring of treatments to reduce or eliminate bacterial burden and promote wound healing has traditionally involved some degree of guesswork, albeit based on skilled judgement. Clinical assessment alone has a low sensitivity in detecting bacteria in chronic wounds $[1,36]$. In the absence of objective evidence of bacterial burden, clinicians must rely solely on proxy signs and symptoms to select a treatment. These treatments vary in cost and complexity and their selection may not demonstrate appropriate antibiotic stewardship. Many advanced therapies are contraindicated for use when high bacterial loads are present [12,37]. Cellular and/or Tissue-based Products (CTPs) for example, have an associated cost of up to USD 2000 to USD 10,000 per application, depending on size, and should not be used in the presence of red or cyan fluorescence in the wound. The use of fluorescence imaging concomitant with advanced therapies such as CTP application may increase their effectiveness and decrease inappropriate CTP application if inadequate wound bed preparation is evident [10,34]. Similarly, the ability to detect bacterial loads at the point-of-care with fluorescence imaging supports clinical judgement when selecting the appropriate intervention to manage bacterial burden in the wound [38-40]. In addition, clinicians can use the real time feedback provided by fluorescence imaging to assess the effectiveness of antiseptic therapy (i.e., dressings and topicals) over time.

Included in this grouping of procedures in Table 4 was the use of imaging for initial assessment of wounds from patients in long term care (LTC) (Figure 3A). Experts working in LTC indicated that immunocompromised elderly patients may not exhibit signs and symptoms of infection; the first evidence of wound infection in these patients is often sepsis $[41,42]$. Therefore, the use of fluorescence imaging at the initial patient encounter may enable wound care providers in this setting to identify at risk patients earlier and reduce risk of invasive infection.

There was a high degree of consensus among experts regarding the procedures and treatments listed in Table 4 that may warrant fluorescence imaging. Of note, for many of these procedures and treatments, experts agreed that fluorescence imaging may be performed before, during and/or after the intervention. Figure 3C,D provide clinical examples of fluorescence imaging used prior to, concurrent with or following mechanical strategies to reduce bacterial burden. Figure 3E,F show use of imaging to provide immediate, actionable information on bacterial burden prior to or following placement of advanced therapies including skin substitutes or grafts and NPWT where high bacterial burden is contraindicated.

The capture of standard images prior to fluorescence images enables clinicians to identify and use anatomical markers or unique characteristics of the wound identified on the standard image (e.g., proximity to medial malleolus or shape of the wound edge) 
as landmarks when interpreting fluorescence images. For example, in Figure 3B, after capturing the fluorescence image, the clinician then reviewed the standard image and compared to the fluorescence images. The irregular shape of the wound edge on the bottom right corner of the wound observed in the standard image was consistent with the pattern of cyan fluorescence (indicative of Pseudomonas aeruginosa) in the fluorescence image. With this information, the clinician was able to narrow down the region of the wound that was most optimal to sample for microbiological analysis. Of note, the range finder function on the device also enables the clinician to image the wound from approximately the same distance as in prior visits.

In instances where red or cyan fluorescence persist after debridement (or other therapies), a wound sample for microbiology may be warranted. More than $87 \%$ of experts agreed that fluorescence imaging may be used to inform the location of sample collected or referral for microbiological analysis. This is consistent with prior studies demonstrating decreased false negative sampling results with fluorescence-informed sampling location [43]. In addition, studies have consistently shown that sampling in regions positive for red or cyan fluorescence will detect elevated levels of bacteria $>95 \%$ of the time $[1,17,18,20,21]$. Sampling for microbiology provides information on the species of bacteria present and antibiotic sensitivities, enabling appropriate selection of an antibiotic when warranted, contributing to disciplined antibiotic stewardship [44,45]. An example of sampling from a region of cyan fluorescence is depicted in Figure 3B.

Table 4. Recommended use of fluorescence imaging for detection of bacterial burden.

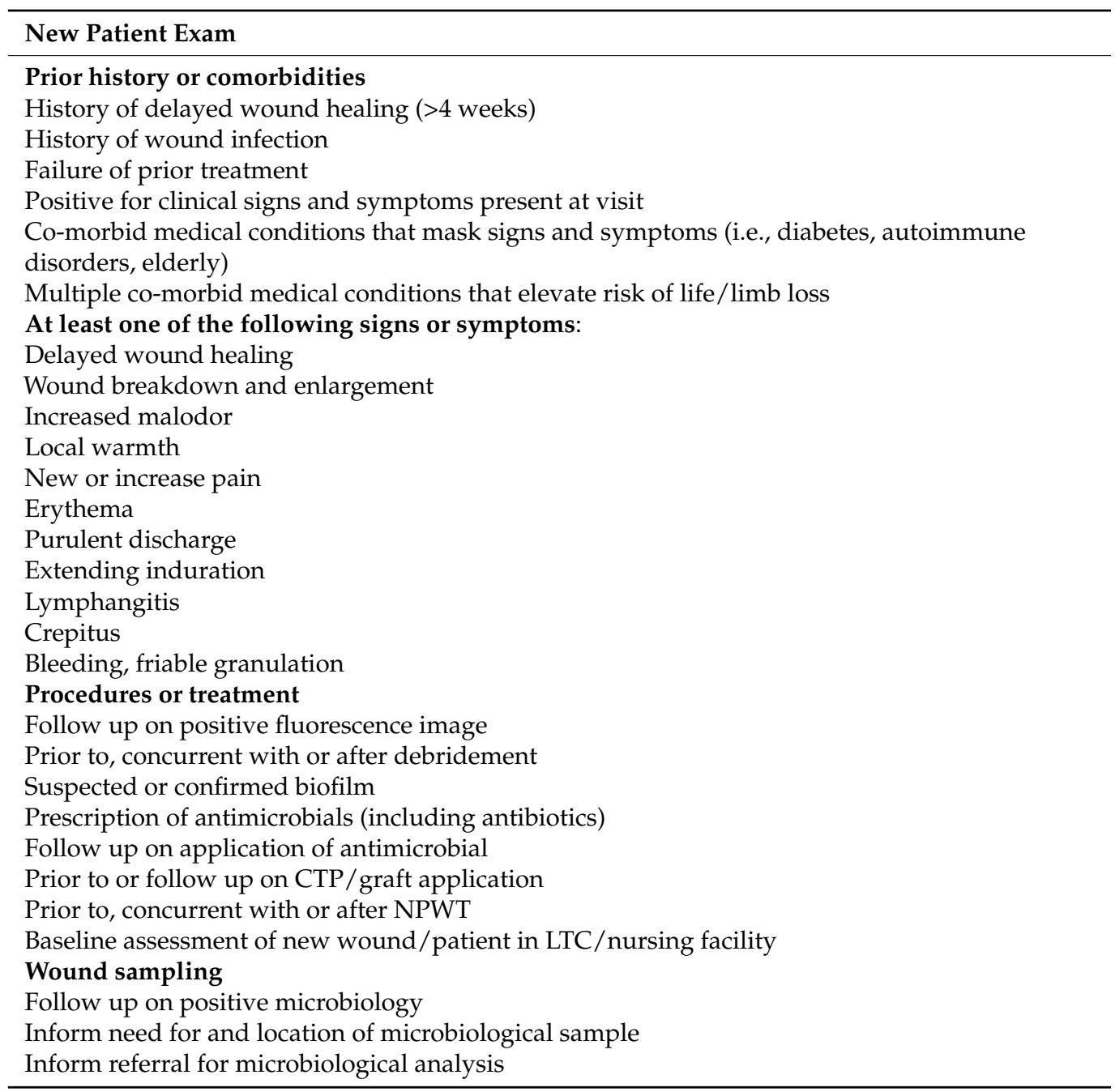




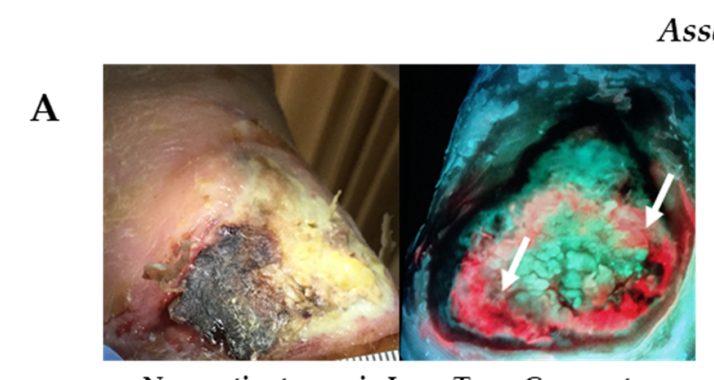

New patient exam in Long Term Care center

\section{Assessment}

Mechanical Strategies to Reduce Bacterial Burden

C

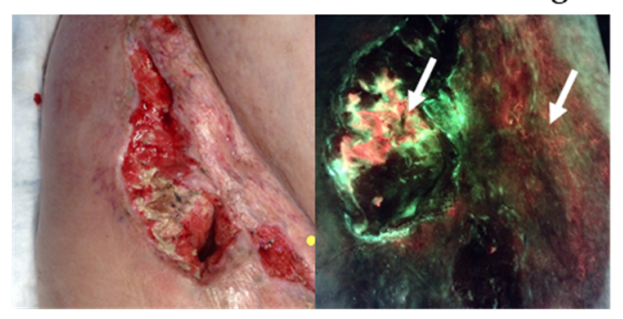

Initial assessment

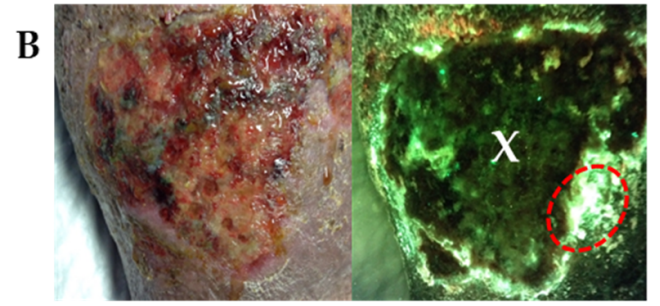

Wound sampling

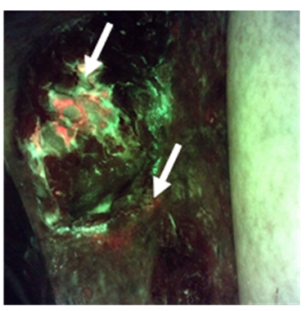

Afterimage informed cleanse

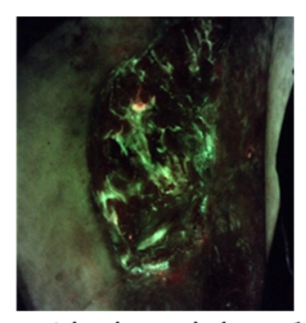

After image informed debridement

D

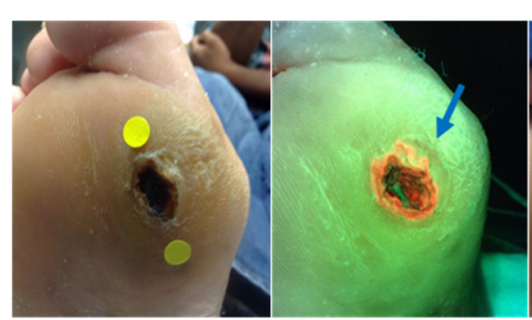

Initial assessment

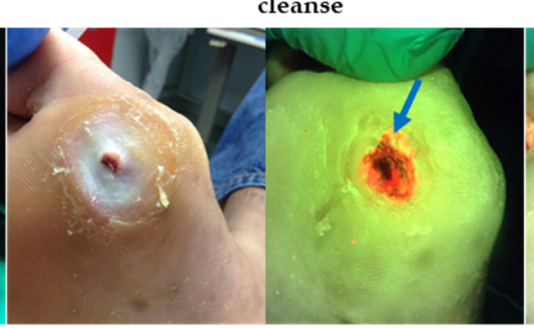

Afterimage informed debridement

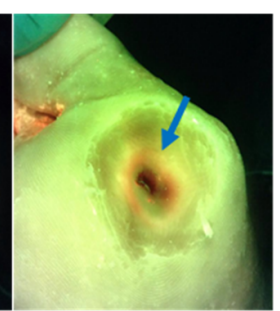

After subsequent image informed debridement

Advanced Therapies Where High Bacterial Burden is Contraindicated

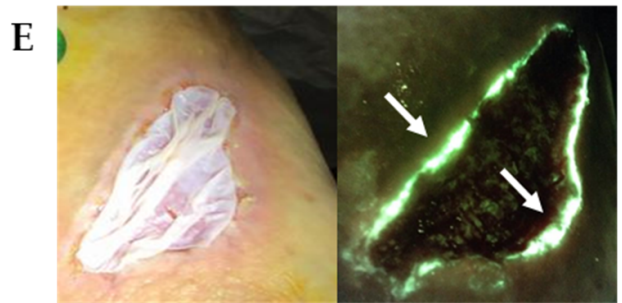

CTP application

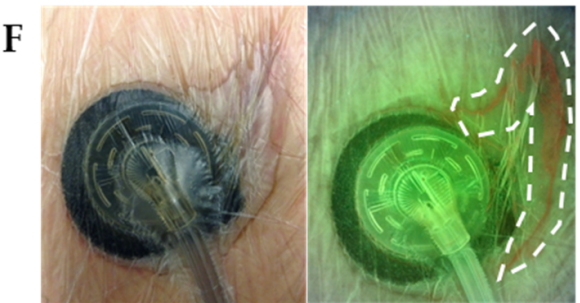

NPWT dressing change

Figure 3. Use of fluorescence imaging across multiple sites of service. (A) Fluorescence images captured at initial assessment of a patient in long term care with a diabetic foot ulcer revealed significant bacterial burden around the wound (white arrows) that changed the plan of treatment put in place for this patient. (B) Images of a venous leg ulcer prompted clinician to collect wound biopsy from region of cyan fluorescence (denoted by red dotted circle), rather than the wound center (denoted by an ' $X$ '); sample was positive for Pseudomonas aeruginosa. (C) Patient with an incisional hip wound following surgery had red fluorescence (white arrows) from bacteria in and around wound. The wound was washed vigorously (HOCL cleanser) and re-imaged. Persistence of red fluorescence prompted additional cleansing and debridement in regions of red fluorescence. Red fluorescence diminished after image-informed mechanical strategies. (D) Red fluorescence from bacteria was detected in a diabetic foot ulcer, highlighted by blue arrow, prompting debridement. Initial debridement revealed a larger amount of bright red fluorescence in the callus tissue surrounding the wound. Additional debridement was performed to remove bacteria laden tissue. (E) A diabetic foot ulcer that had previously received multiple CTP applications underwent fluorescence imaging prior to application of another CTP. Images revealed the presence of cyan fluorescence (white arrows) indicative of Pseudomonas aeruginosa around the wound edge; CTP application was withheld until cyan signal was eliminated. (F) NPWT was applied to an appendectomy abscess. Fluorescence images were captured during a dressing change and indicated presence of red fluorescence around the wound (denoted by dashed white dotted line). Imaging informed mechanical bacterial removal prior to placement of a new dressing. CTP, cellular tissue product; NPWT, negative pressure wound therapy. 


\subsubsection{Recommended Frequency of Fluorescence Imaging}

One of the most common questions that arise when clinicians first adopt this diagnostic imaging technology is "how often should I image my patients?" Unlike other imaging procedures (e.g., X-rays) that pose a risk to the patient with prolonged exposure, fluorescence imaging uses a safe, visible violet light to detect bacteria; as such, imaging can be performed repeatedly without any detriment to patients. Typically, clinicians capture a fluorescence image at the initial patient encounter (baseline) to determine the status of the wound and identify additional tests or treatments that may be needed. At subsequent patient encounters, $>93.5 \%$ of experts agreed that fluorescence imaging should be performed no more than weekly, unless otherwise clinically indicated (Table 5). This frequency was determined based on the understanding that many prescribed interventions aimed at targeting bacterial burden and promoting wound healing (e.g., antimicrobial dressings) take days or weeks to observe an effect. This frequency also matched the typical frequency at which patients visit wound care centers for follow up visits. The time intervals may decrease (i.e., more than once a week) or increase (i.e., evaluations every 2-4 weeks), depending on the progression of the clinical status. The core panel emphasized the importance of medical necessity and use of clinical judgement to avoid unnecessary procedures or treatments while ensuring that patients receive the optimal care needed to heal their wounds.

Table 5. Recommended frequency of fluorescence imaging.

\section{Recommended Frequency of Fluorescence Imaging.}

Fluorescence imaging may be performed on wounds at baseline, during the first 4 weeks and/or if an increase in wound size is observed

Fluorescence imaging may be performed weekly on patients that meet criteria for imaging (e.g., clinical signs and symptoms) If a wound is positive for fluorescence, fluorescence imaging is performed no more than on a weekly basis; unless otherwise indicated by development or change in symptoms

\subsection{Reported Impact of Fluorescence Imaging on Treatment Planning $\mathcal{E}$ Wound Outcomes}

This consensus provided a unique opportunity to receive feedback from a group of wound care experts with significant experience implementing fluorescence imaging into their clinical care pathway. To better appreciate the potential impact of the objective information provided by fluorescence imaging, several questions were included in the Delphi, assessing the impact of fluorescence imaging on treatment planning and wound outcomes. Most experts (87.6\%) indicated that the objective information provided by fluorescence imaging changed their treatment plans in a significant number of cases (Figure 4A). These findings are consistent with results from a clinical study of 350 patients in which changes to treatment plans following capture and review of fluorescence images were observed in $70 \%$ of patients [1]. A similar response was observed when experts were asked to report the impact of fluorescence imaging on rates of wound microbiological sampling. $83 \%$ of experts reported that fluorescence imaging reduced their rate of microbiological sampling, of which 56\% reported a significant reduction (>25\%) (Figure 4B). Panelists agreed that there will be times when sampling information is critical, and that fluorescence imaging cannot replace sampling in terms of confirming antibiotic susceptibilities and speciation. The reduced rate of sampling reported by experts indicates that many felt confident in using fluorescence imaging information to monitor changes in bacterial burden over time.

Included in the Delphi were questions on the reported impact of fluorescence imaging on patients' wound outcomes. $96 \%$ of experts reported that, in their experience, imaging led to improved wound healing (Figure 4C). When asked about rates of amputation, $78 \%$ of experts reported reduced rates of amputation with use of fluorescence imaging (Figure 4D). These reports are in line with recent studies evaluating the impact of fluorescence imaging on wound outcomes. A 2-year retrospective analysis of 229 foot ulcers reported a $23 \%$ increase in wound healing rates with incorporation of fluorescence imaging. These improved 
wound healing rates were attributed to earlier bacterial detection and more thorough debridement performed at the point-of-care, using objective fluorescence information [23]. Multiple recent randomized controlled trials (RCTs) have also demonstrated substantial improvements (>200\%) in 12-week wound healing rates when fluorescence imaging is incorporated into standard of care [46,47]. In these RCTs, improvements in wound healing rates occurred without increases in antibiotic prescribing and without addition of CTPs, suggesting a substantial decrease in cost of care. These findings suggest that by following a workflow as described in Figure 2, patients may receive more optimal treatment from their wound care providers, contributing to improved outcomes as described in Figure 4.

A

\section{B}

\section{"In my experience, fluorescence imaging has reduced my rate of wound} microbiological analysis"

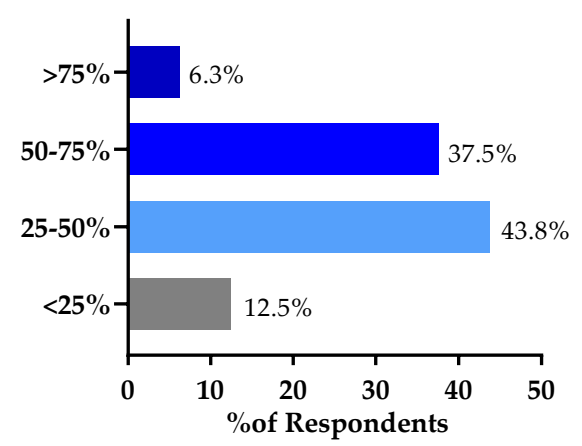

C

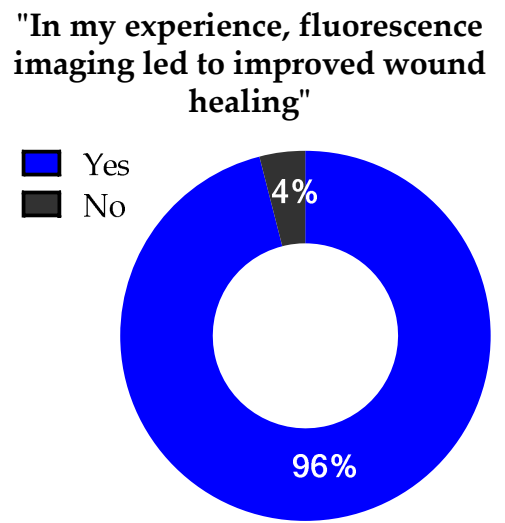

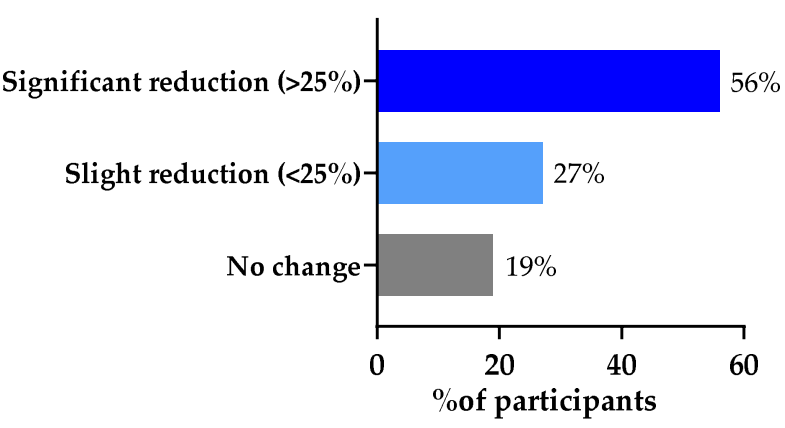

D

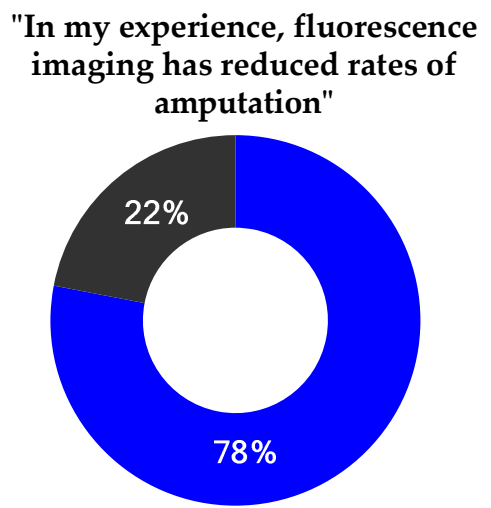

Figure 4. Impact of fluorescence imaging of bacteria on treatment protocols and wound outcomes. A total of 31 participants provided responses. (A) Percent of Delphi participants reporting changes in treatment plans based on fluorescence imaging. (B) Percent of participants reporting reduction in wound sampling for microbiological analysis because of point-of-care fluorescence imaging information. (C) Percent of participants indicating improvements in wound healing due to use of fluorescence imaging. (D) Percent of participants indicating reduced rates of amputation due to use of fluorescence imaging.

\section{Conclusions}

Consensus on guidelines for the use of fluorescence imaging was obtained through a two-round Delphi process. The final product includes a series of guidelines (Tables 2-4) and a clinical workflow (Figure 2) that provides a framework for multiple stakeholders including clinicians, medical, surgical, and wound care societies, policy makers and regulatory bodies. Evidence indicates that use of consensus guidelines improves the quality of healthcare provided by supporting evidence-based, best-practice care. Furthermore, development of a clinical workflow algorithm provides a stepwise sequence that improves the consistency and coordination of care across the wound care continuum [25].

The topics included in the Delphi consensus address many common concerns that new users encounter when adopting this technology. For example, information on clinical 
indications and frequency of imaging can aid clinicians in understanding the medical necessity needed to perform this procedure. Statement development was based on peerreviewed publications in addition to the clinical experience of a diverse panel of wound care experts familiar with the fluorescence imaging procedure. There was a high level of agreement $(>85 \%)$ among wound care experts familiar with the fluorescence imaging procedure (well above the typical threshold for most consensus statements), indicating a high level of support for the adoption of these clinical indications. A summary of guidelines based on the result of this Delphi consensus is listed in Table 6.

Table 6. Summary of guidelines based on Delphi consensus.

Summary of Guidelines for Fluorescence Imaging

Training is needed to effectively perform fluorescence imaging.

E.g., how to position the patient and device, how to interpret images

The clinical workflow for fluorescence imaging compliments and is in addition to clinical wound assessment and treatment.

Clinical judgement should be used to apply fluorescence information to treatment decision making.

Medical history, comorbidities and signs of infection may inform the medical necessity for fluorescence imaging.

E.g., history of delayed wound healing, presence of diabetes, detection of pain or malodor

Fluorescence imaging may be performed prior to, concurrent with, or following many common procedures and therapies in

wound care.

E.g., Debridement, CTP application, prescription of antimicrobials, wound sampling

Fluorescence imaging should be performed no more than weekly, unless otherwise indicated by medical necessity.

The high level of agreement among experts demonstrates the utility and value of this diagnostic technology in the management of chronic wounds. Use of this imaging procedure enables healthcare providers to rapidly detect elevated levels of bacteria and develop an effective treatment plan while at the same time avoiding the use of advanced therapies when they are contradicted. In addition to improving patient outcomes, this technology has high potential to reduce the overuse of antimicrobials and reduce healthcare costs for both patients and health systems.

Author Contributions: Conceptualization A.R.O., C.A., R.A., J.H., M.K., M.M., T.E.S.; data acquisition, T.E.S.; writing-original draft preparation, T.E.S.; writing-review and editing, A.R.O., C.A., J.H., M.K., M.M. All authors have read and agreed to the published version of the manuscript.

Funding: This research received no external funding.

Institutional Review Board Statement: Not applicable.

Informed Consent Statement: Informed consent was obtained from all subjects whose medical images are shown.

Conflicts of Interest: The authors declare no conflict of interest.

\section{References}

1. Le, L.; Baer, M.; Briggs, P.; Bullock, N.; Cole, W.; DiMarco, D.; Hamil, R.; Harrell, K.; Kasper, M.; Li, W.; et al. Diagnostic Accuracy of Point-of-Care Fluorescence Imaging for the Detection of Bacterial Burden in Wounds: Results from the 350-Patient Fluorescence Imaging Assessment and Guidance Trial. Adv. Wound Care 2021, 10, 123-136. [CrossRef] [PubMed]

2. Serena, T.E.; Harrell, K.; Serena, L.; Yaakov, R.A. Real-time bacterial fluorescence imaging accurately identifies wounds with moderate-to-heavy bacterial burden. J. Wound Care 2019, 28, 346-357. [CrossRef]

3. Reddy, M.; Gill, S.S.; Wu, W.; Kalkar, S.R.; Rochon, P.A. Does this patient have an infection of a chronic wound? JAMA 2012, 307, 605-611. [CrossRef] [PubMed]

4. Lantis, J.C.; Marston, W.A.; Farber, A.; Kirsner, R.S.; Zhang, Y.; Lee, T.D.; Cargill, D.I.; Slade, H.B. The influence of patient and wound variables on healing of venous leg ulcers in a randomized controlled trial of growth-arrested allogeneic keratinocytes and fibroblasts. J. Vasc. Surg. 2013, 58, 433-439. [CrossRef]

5. Lookingbill, D.P.; Miller, S.H.; Knowles, R.C. Bacteriology of chronic leg ulcers. Arch. Dermatol. 1978, 114, 1765-1768. [CrossRef]

6. Majewski, W.; Cybulski, Z.; Napierala, M.; Pukacki, F.; Staniszewski, R.; Pietkiewicz, K.; Zapalski, S. The value of quantitative bacteriological investigations in the monitoring of treatment of ischaemic ulcerations of lower legs. Int. Angiol. 1995, 14, 381-384. [PubMed] 
7. Martín, S.; Pérez, A.; Aldecoa, C. Sepsis and Immunosenescence in the Elderly Patient: A Review. Front. Med. 2017, 4, 20. [CrossRef]

8. Sen, C.K.; Gordillo, G.M.; Roy, S.; Kirsner, R.; Lambert, L.; Hunt, T.K.; Gottrup, F.; Gurtner, G.C.; Longaker, M.T. Human skin wounds: A major and snowballing threat to public health and the economy. Wound Repair Regen. 2009, 17, 763-771. [CrossRef]

9. Nussbaum, S.R.; Carter, M.J.; Fife, C.E.; DaVanzo, J.; Haught, R.; Nusgart, M.; Cartwright, D. An Economic Evaluation of the Impact, Cost, and Medicare Policy Implications of Chronic Nonhealing Wounds. Value Health 2018, 21, 27-32. [CrossRef]

10. Caldwell, M.D. Bacteria and Antibiotics in Wound Healing. Surg. Clin. N. Am. 2020, 100, 757-776. [CrossRef] [PubMed]

11. Browne, A.C.; Vearncombe, M.; Sibbald, R.G. High bacterial load in asymptomatic diabetic patients with neurotrophic ulcers retards wound healing after application of Dermagraft. Ostomy Wound Manag. 2001, 47, 44-49.

12. Hogsberg, T.; Bjarnsholt, T.; Thomsen, J.S.; Kirketerp-Moller, K. Success rate of split-thickness skin grafting of chronic venous leg ulcers depends on the presence of Pseudomonas aeruginosa: A retrospective study. PLoS ONE 2011, 6, e20492. [CrossRef] [PubMed]

13. Ai-Jalodi, O.; Sabo, M.; Patel, K.; Bullock, N.; Serena, L.; Breisinger, K.; Serena, T.E. Efficacy and safety of a porcine peritoneumderived matrix in diabetic foot ulcer treatment: A pilot study. J. Wound Care 2021, 30, S18-S23. [CrossRef]

14. Esper, A.M.; Moss, M.; Lewis, C.A.; Nisbet, R.; Mannino, D.M.; Martin, G.S. The role of infection and comorbidity: Factors that influence disparities in sepsis. Crit. Care Med. 2006, 34, 2576-2582. [CrossRef]

15. Armstrong, D.G.; Swerdlow, M.A.; Armstrong, A.A.; Conte, M.S.; Padula, W.V.; Bus, S.A. Five year mortality and direct costs of care for people with diabetic foot complications are comparable to cancer. J. Foot Ankle Res. 2020, 13, 16. [CrossRef]

16. Rennie, M.Y.; Dunham, D.; Lindvere-Teene, L.; Raizman, R.; Hill, R.; Linden, R. Understanding Real-Time Fluorescence Signals from Bacteria and Wound Tissues Observed with the MolecuLight i:X(TM). Diagnostics 2019, 9, 22. [CrossRef] [PubMed]

17. Hill, R.; Woo, K. A Prospective Multisite Observational Study Incorporating Bacterial Fluorescence Information Into the UPPER/LOWER Wound Infection Checklists. Wounds 2020, 32, 299-308.

18. Hurley, C.M.; McClusky, P.; Sugrue, R.M.; Clover, J.A.; Kelly, J.E. Efficacy of a bacterial fluorescence imaging device in an outpatient wound care clinic: A pilot study. J. Wound Care 2019, 28, 438-443. [CrossRef] [PubMed]

19. Jones, L.M.; Dunham, D.; Rennie, M.Y.; Kirman, J.; Lopez, A.J.; Keim, K.C.; Little, W.; Gomez, A.; Bourke, J.; Ng, H.; et al. In vitro detection of porphyrin-producing wound bacteria with real-time fluorescence imaging. Future Microbiol. 2020, 15, 319-332. [CrossRef]

20. Raizman, R.; Little, W.; Smith, A.C. Rapid Diagnosis of Pseudomonas aeruginosa in Wounds with Point-Of-Care Fluorescence Imaing. Diagnostics 2021, 11, 280. [CrossRef]

21. Rennie, M.Y.; Lindvere-Teene, L.; Tapang, K.; Linden, R. Point-of-care fluorescence imaging predicts the presence of pathogenic bacteria in wounds: A clinical study. J. Wound Care 2017, 26, 452-460. [CrossRef] [PubMed]

22. Hill, R.; Rennie, M.Y.; Douglas, J. Using Bacterial Fluorescence Imaging and Antimicrobial Stewardship to Guide Wound Management Practices: A Case Series. Ostomy Wound Manag. 2018, 64, 18-28. [CrossRef]

23. Price, N. Routine fluorescence imaging to detect wound bacteria reduces antibiotic use and antimicrobial dressing expenditure while improving healing rates: Retrospective analysis of 229 foot ulcers. Diagnostics 2020, 10, 927. [CrossRef] [PubMed]

24. Cole, W.; Coe, S. Use of a bacterial fluorescence imaging system to target wound debridement and accelerate healing: A pilot study. J. Wound Care 2020, 29, S44-S52. [CrossRef]

25. Eubank, B.H.; Mohtadi, N.G.; Lafave, M.R.; Wiley, J.P.; Bois, A.J.; Boorman, R.S.; Sheps, D.M. Using the modified Delphi method to establish clinical consensus for the diagnosis and treatment of patients with rotator cuff pathology. BMC Med. Res. Methodol. 2016, 16, 56. [CrossRef]

26. Kim, P.J.; Attinger, C.E.; Steinberg, J.S.; Evans, K.K.; Lehner, B.; Willy, C.; Lavery, L.; Wolvos, T.; Orgill, D.; Ennis, W.; et al. Negative-pressure wound therapy with instillation: International consensus guidelines. Plast. Reconstr. Surg. 2013, 132, 1569-1579. [CrossRef]

27. Russell, D.; Atkin, L.; Betts, A.; Dowsett, C.; Fatoye, F.; Gardner, S.; Green, J.; Manu, C.; McKenzie, T.; Meally, H.; et al. Using a modified Delphi methodology to gain consensus on the use of dressings in chronic wounds management. J. Wound Care 2018, 27, 156-165. [CrossRef] [PubMed]

28. Cutting, K.F.; White, R.J.; Mahoney, P. Developing clinical criteria for wound infection-A delphi approach: 680. J. Wound Ostomy Cont. Nurs. 2005, 32, S26. [CrossRef]

29. Hohmann, E.; Brand, J.C.; Rossi, M.J.; Lubowitz, J.H. Expert Opinion Is Necessary: Delphi Panel Methodology Facilitates a Scientific Approach to Consensus. Arthroscopy 2018, 34, 349-351. [CrossRef]

30. Hasson, F.; Keeney, S.; McKenna, H. Research guidelines for the Delphi survey technique. J. Adv. Nurs. 2000, 32, $1008-1015$.

31. Keeney, S.; Hasson, F.; McKenna, H. Consulting the oracle: Ten lessons from using the Delphi technique in nursing research. J. Adv. Nurs. 2006, 53, 205-212. [CrossRef]

32. MacLeod, B.G.; Klarich, C.S.; Wessman, L.L.; Gaddis, K.P.; Konstantinov, N.K.; Wubben, A.; Melin, M.M. Novel Diagnostic Fluorescence Imaging and Therapeutic Considerations for the Treatment of Pyoderma Gangrenosum. Adv. Ski. Wound Care 2021, in press.

33. Raizman, R. Bacterial fluorescence imaging guides wound cleaning and facilitates patient education in pilonidal sinus wound care patients. In Proceedings of the European Wound Management Association, Amsterdam, The Netherlands, 3-6 May 2017. 
34. Wu, Y.C.; Smith, M.; Chu, A.; Lindvere-Teene, L.; Starr, D.; Tapang, K.; Shekhman, R.; Wong, O.; Linden, R.; DaCosta, R.S. Handheld fluorescence imaging device detects subclinical wound infection in an asymptomatic patient with chronic diabetic foot ulcer: A case report. Int. Wound J. 2016, 13, 449-453. [CrossRef]

35. Xu, L.; McLennan, S.V.; Lo, L.; Natfaji, A.; Bolton, T.; Liu, Y.; Twigg, S.M.; Yue, D.K. Bacterial load predicts healing rate in neuropathic diabetic foot ulcers. Diabetes Care 2007, 30, 378-380. [CrossRef] [PubMed]

36. Gardner, S.E.; Hillis, S.L.; Frantz, R.A. Clinical signs of infection in diabetic foot ulcers with high microbial load. Biol. Res. Nurs. 2009, 11, 119-128. [CrossRef]

37. Zekri, A.; King, W. Success of skin grafting on a contaminated recipient surface. Eur. J. Plast. Surg. 1995, 18, 40-42. [CrossRef]

38. Aung, B. Can Fluorescence Imaging Predict the Success of CTPs for Wound Closure and Save Costs? Today's Wound Clin. 2019, 13, 22-25.

39. Raizman, R. Fluorescence imaging guided dressing change frequency during negative pressure wound therapy: A case series. J. Wound Care 2019, 28, S28-S37. [CrossRef]

40. Serena, T. A Paradigm Shift in Chronic Wound Assessment: Incorporating Bacterial Fluorescence Imaging into Standard of Care. In Photonic Diagnosis, Monitoring, Prevention, and Treatment of Infections and Inflammatory Diseases; SPIE: San Fransico, CA, USA, 2020; Volume 112230F. [CrossRef]

41. Girard, T.D.; Ely, E.W. Bacteremia and sepsis in older adults. Clin. Geriatr. Med. 2007, 23, 633-647. [CrossRef]

42. Nasa, P.; Juneja, D.; Singh, O. Severe sepsis and septic shock in the elderly: An overview. World J. Crit. Care Med. 2012, 1, 23-30. [CrossRef]

43. Raizman, R.; Dunham, D.; Lindvere-Teene, L.; Jones, L.M.; Tapang, K.; Linden, R.; Rennie, M.Y. Use of a bacterial fluorescence imaging device: Wound measurement, bacterial detection and targeted debridement. J. Wound Care 2019, 28, 824-834. [CrossRef]

44. Bonham, P.A. Swab cultures for diagnosing wound infections: A literature review and clinical guideline. J. Wound Ostomy Cont. Nurs. 2009, 36, 389-395. [CrossRef]

45. Rondas, A.A.; Schols, J.M.; Halfens, R.J.; Stobberingh, E.E. Swab versus biopsy for the diagnosis of chronic infected wounds. Adv. Ski. Wound Care 2013, 26, 211-219. [CrossRef]

46. Rahma, S.; Woods, J.; Nixon, J.; Brown, S.; Russel, D. The use of Point-of-Care Bacterial Autofluorescence Imaging in the Management of Diabetic Foot Ulcers; A Pilot Randomised Controlled Trial. In Proceedings of the Symposium on Advanced Wound Care Virtual, Online, 4-6 November 2020.

47. Oropallo, A.; Gawlik, S.; Vayser, D. 12-week RCT Evaluating Impact of Routine Fluorescence Imaging of Bacteria on DFU Healing Rates. In Proceedings of the Symposium on Advanced Wound Care Virtual, Online, 10-14 May 2021. 